nor risk factors for HEV. Although HEV serology was initially equivocal, IgG and IgM later became positive with detectable PCR. His ALT normalised and HEV-PCR became undetectable four weeks later.

Discussion/conclusion HEV appears to be a self-limiting-asymptomatic illness in HIV+ MSM with good CD4 counts. HEV may be sexually transmitted in populations with increasing STI rates. HEV should be considered a potential cause of elevated liver enzymes in HIV+ patients.

\section{P35 ARE TESTICULAR MIXED GERM CELL TUMOURS ASSOCIATED WITH HEPATITIS C(HCV) IN HIV INFECTED MEN WHO HAVE SEX WITH MEN?}

David Atefi* , Daniel Richardson. BSUH NHS Trust, Brighton, UK

\subsection{6/sextrans-2015-052126.79}

Background/introduction HCV infection and testicular germ-cell tumours are indicator diseases for HIV-testing in BASHH-guidelines. There is little data on the association of testicular tumours in MSM with HIV.

Aim(s)/objectives We describe 2 MSM with treated HIV-Hepatitis $\mathrm{C}$ co-infection who were both subsequently diagnosed with mixed-germ-cell testicular tumours.

Case details Patient-1 is a 51-year-old MSM, diagnosed with HIV in 2004 on Atripla since 2010. In May 2012, routine ALT $=186$ and positive HCV-RNA (genotype 1). This was treated with 48-weeks of pegylated-interferon/ribavirin. He had a sustained-viral-response (SVR). Two years later, he presented to the STI-clinic with a four month history of testicular swelling. Ultrasound showed this to be likely malignant infiltration, AFP = $2484, \mathrm{LDH}=426, \mathrm{HCG}=5.9$. After orchidectomy, histology demonstrated mixed germ cell tumour. He is in clinical/radiological remission. Patient-2 is a 41-year-old MSM diagnosed with HIV in 2004. In 2007 he received IL-2 in a clinical trial. In both 2008 and 2012 routine ALT $=918,505$ respectively and HCVRNA was positive (genotype $2 / 3$ )(genotype 1 ). HCV was treated with pegylated-interferon/ribavirin both times with SVR. Antiretrovirals (Atripla) were started in 2012. That year, he presented with an E-Coli-UTI and testicular swelling. Ultrasound/ orchidectomy found a mixed germ cell testicular tumour. Tumour markers were AFP $=16.5, \mathrm{LDH}=376, \mathrm{HCG}=16.5$. He was treated with orchidectomy, bleomycin/etoposide/cisplatin and is in radiological/clinical remission.

Discussion/conclusion HIV infection and hepatitis C treatment are immunosuppressive and are potential causative factors in these HIV-MSM testicular germ-cell tumours. Early investigation of testicular swellings in men with HIV-Hepatitis C is important.

\section{P36 AUTOIMMUNE HEPATITIS IN A PATIENT WITH HIV AND HEPATITIS B CO-INFECTION ALONG WITH LATENT TB: A THERAPEUTIC DILEMMA}

Nisha Pal, Mamatha Odhuru*, Noreen Desmond. Berkshire Healthcare Foundation Trust, Garden Clinic, Slough, Berkshire, UK

10.1136/sextrans-2015-052126.80

Background/introduction Liver disease is an important cause of morbidity and mortality in patients infected with HIV infection. Abnormal liver function tests are frequently encountered in these patients and often attributed to HAART. Autoimmune Hepatitis is a rare disease with unclear pathogenesis; several viruses have been proposed to act as triggering agents for the inflammatory process of the disease.

Case presentation We present a 46 year old afro Caribbean gentleman who presented with lethargy, weight loss and jaundice. He was diagnosed to be co-infected with HIV and Hepatitis B with a positive autoimmune screen. His persistently elevated liver enzymes warranted a liver biopsy which revealed interface hepatitis, necrosis with lymphocytes and plasma cell infiltrates with variable degree of fibrosis. A picture difficult to interpret in the light of HIV and Hepatitis B infections. His persistently high ALT made it challenging to initiate antiretroviral therapy and the need for steroids to suppress the autoimmune Hepatitis raised a concern regarding the reactivation of the latent $\mathrm{TB}$ infection as diagnosed by a positive IGRA test. A review of literature revealed 12 cases of HIV with AIH, but none co-infected with Hepatitis B.

Conclusion There are no clear guidelines for management of autoimmune Hepatitis in HIV and treatment is with immunosuppressive agents. A multidisciplinary approach helped in the management of this gentlemen who now stable on antiretroviral therapy and tapering doses of steroids, along with chemoprophylaxis against latent $\mathrm{TB}$.

\section{P37 SEXUALLY ACQUIRED SALMONELLA TYPHI URINARY TRACT INFECTION}

Sally Wielding*, Gordon Scott. Chalmers Centre, NHS Lothian, Edinburgh, UK

10.1136/sextrans-2015-052126.81

Case report A 22 year old MSM was diagnosed HIV positive with a CD4 cell count was 475 cells/mm3 (35\%). He suffered urinary symptoms and Salmonella typhi was isolated from urine culture. He recalled a self-limiting afebrile diarrhoeal illness 2 weeks earlier. Stool and blood cultures were negative. He completed a one-week course of ciprofloxacin with subsequently negative cultures. He had no past medical history or significant travel history. He reported unprotected anal intercourse one month before HIV diagnosis, and protected anal intercourse with several partners since diagnosis, but no other infections have been reported locally. All named contacts have declined testing.

Discussion The most common manifestation of S.typhi infection is typhoid fever. Most cases in the developed world have been acquired through faeco-oral transmission in endemic areas. Haematogenous dissemination can be widespread and more severe among the immunocompromised. Death ensues in up to $32 \%$. Infection of the genitourinary system is rare. Cases reported have a background of urinary tract abnormalities, invariably with blood and/or stool culture positivity. There are no cases in the literature of sexually acquired S.typhi UTI. Infections were acquired through oro-anal contact and pathogen ingestion. None had UTI. Our patient had repeatedly negative blood and stool cultures, reducing the likelihood that this was a disseminated infection leading to UTI, and raising the possibility that the route of infection was though insertive anal intercourse with direct urethral inoculation with S.typhi. Unfortunately partner notification has not identified an infected sexual contact to add further weight to this theory. 